\title{
BASES DE UN SISTEMA DE GESTIÓN DE CALIDAD PARA UN CENTRO DE TÉCNICAS DE REPRODUCCIÓN ASISTIDA
}

\section{The basis for a quality management system for an assisted reproduction technique centre}

Jaime Saavedra-Saavedra, M.D.*

Recibido: marzo 15/06 - Revisado: mayo 23/06 - Aceptado: Junio 5/06

\section{RESUMEN}

El propósito de este informe es revisar aquellos factores que se deberían considerar en la evaluación de calidad total de un centro de técnicas de reproducción asistida.

Metodología: se revisan las definiciones de calidad, gestión de calidad y calidad en centros de reproducción. Se revisa la percepción del cliente sobre calidad en estos centros y se describen los indicadores aceptados por las entidades internacionales de la subespecialidad.

Resultados: se hace una aproximación crítica a estos indicadores con la descripción de la acreditación de este centro como ejemplo del proceso a seguir, se brindan los indicadores de la entidad.

Palabras clave: calidad de la atención en salud, normas ISO 9001:2000, tasa de embarazo, atención al cliente.

\section{SUMMARY}

This paper is aimed at examining the factors which should be considered when evaluating an assisted

\footnotetext{
Profesor Titular Departamento de Ginecología y Obstetricia, Universidad Del Valle - Cali Colombia.

Director del Centro de Biomedicina Reproductiva -Fecundar Cali - Colombia

Cra 40 No 5B-105 PBX:5534300

Correo electrónico: fecundar@colombianet.net
}

reproduction centre's overall quality. Normal methods for evaluating quality were reviewed. Many state regulating institutions around the world have become centred just on pregnancy rates, which could lead to erroneous conclusions if other essential factors are not taken in count, such as multiple pregnancy, ovarian hyper-stimulation, customer satisfaction, suitable laboratory evaluation and applying clinical protocols. This article describes how our institution was accredited as a model for such process. Methods and procedures for improving quality are essential in all business models, including those pertaining to assisted reproduction centres. ISO 9001 is applied in our institution when evaluating and improving the service provided for our patients.

Key words: quality of health care, norms ISO 9001:2000, pregnancy rates, client attention.

\section{INTRODUCCIÓN}

En el año 2004, Fecundar, Centro de Reproducción Asistida de la ciudad de Cali - Colombia, tomó la decisión de evaluar su programa, determinar las características de sus clientes y sus necesidades, así como los resultados de los procedimientos que realizaba. Las observaciones anteriores nos llevaron a un largo proceso para implementar el Sistema de 
Gestión de Calidad ISO-9001 Versión 2000, esto condujo a un rediseño en la política, la misión y visión de nuestra institución. En julio de 2005 obtuvimos la certificación como el primer centro en Colombia que cumplía con los máximos estándares de calidad en los todos los procesos relacionados con las técnicas de reproducción asistida.

Las herramientas y principios utilizados para medir calidad de un Centro de Fertilización In Vitro (FIV) son muy similares a las de muchas otras industrias. A pesar de que el estándar internacional para la medición de calidad es ISO 9000 tiene ya 5 años muy pocos centros de FIV han aplicado la norma. ${ }^{1}$

¿Que es ISO? Es la Organización Internacional de Estandarización, estas siglas se derivan del termino griego isos, que significa igual, fue fundada en Ginebra, Suiza en 1946. El documento ISO 9000 se publicó por primera vez en 1987 y consiste en realidad en cinco normas relacionadas entre sí, numeradas del 9000 al 9004 (9000, 9001, 9004, 9011) las que sin embargo van en aumento. ${ }^{2}$

ISO 9000 impone a las empresas la obligación de documentar sus procesos, los sistemas de calidad, garantizar que todos los empleados conozcan y sigan los lineamientos del documento, que el sistema de calidad sea continuamente vigilado y revisado por medio de auditorias internas o externas y por último se proceda efectivamente en la realización de los cambios necesarios.

El propósito de esta publicación es examinar aquellos factores que se deberían considerar para determinar la calidad total de un centro de Técnicas de Reproducción Asistida.

\section{MATERIALES Y MÉTODOS}

Definiciones conceptuales acerca de la calidad:

Calidad se ha definido como: "la satisfacción de las expectativas del cliente". Supone entender de manera clara y sin ambigüedades las necesidades del cliente. Esta definición ha presentado modificaciones, por ejemplo "Calidad es el suministro de bienes que no regresan, a clientes que si lo hacen”. Aunque hay cierta sutileza en esta definición, incorpora un axioma comercial. $^{3}$

- Otras definiciones de calidad son: La correspondencia de lo que se desea con lo que se consigue.

- La expectativa versus la satisfacción de la necesidad.

- Dar al cliente lo que desea hoy; a un precio que pueda pagar; a un costo que podamos soportar; una y otra vez y otra vez; y darle algo aún mejor mañana.

Gestión de calidad: es un servicio que recopila información. con base en los datos que se dispone de cada proceso y cada actividad de servicio, extrae aquello que tiene más significado de lo que tiene menos significado, mediante ese proceso los datos empiezan a controlar el comportamiento futuro de ese proceso con miras a una mayor satisfacción del cliente.

La calidad desde el punto de vista de los servicios de reproducción humana: Los recursos disponibles que tiene un centro de reproducción humana son cuatro: materias primas, equipos especiales, tiempo y gente. El objetivo del director científico de una unidad de reproducción humana consiste en hacer el mejor uso del tiempo y de tecnología por medio de las competencias de sus empleados, con el fin de lograr el objetivo del servicio: la consecución de un embarazo, el cual debe terminar con un niño en casa.

¿Quién es el responsable de la calidad? La responsabilidad por la calidad pertenece al proceso y a quienes cuya administración incluye todo el proceso de generación de resultados. Colectivamente son los guardianes de los recursos, cuya finalidad es la generación de resultados agregando valor a través de la actividad llamada trabajo. ${ }^{3} \mathrm{El}$ argumento para la mejor definición de calidad en nuestro campo es la utilización de procesos que sean estandarizados. La tarea del departamento de calidad es evaluar la capacidad del proceso, equilibrar el sube y baja en uno de cuyos extremos están las necesidades de los clientes, mientras en 
el otro se sitúa la capacidad medida del proceso para satisfacer esas necesidades.

\section{Cómo percibe el cliente la calidad en los servicios de reproducción}

$\mathrm{Al}$ evaluar los factores más relevantes que afectan la decisión de los pacientes en la escogencia de la clínica para el tratamiento de su problema de fertilidad, se encontró primero que ellos están bien informados y conocen el mercado antes de tomar la decisión y obtienen la información de muchas fuentes, incluyendo el Internet. Segundo, el factor más relevante en la toma de la decisión es las tasas de éxito (p.e. tasas de parto de la clínica) y el tercer factor es la recomendación del médico general o ginecólogo. Otros factores como son el costo del tratamiento, la opinión de amigos y familiares así como la distancia al hogar no fueron importantes. ${ }^{4}$

\section{¿Qué entidades normatizan la calidad de la Fertilización In Vitro (FIV)?}

El establecimiento de estos controles ha sido resultado de presiones del público y de políticos para que los centros de FIV reporten sus resultados. Se deduce, que esto es un intento para tener una medida de la calidad en la forma de tasas de embarazo. Estas funciones son realizadas en Estados Unidos por la SART (Society of Reproductive Technology), en Francia por la FIVNAT (French In Vitro National) y en Inglaterra por la HFEA (Human Fertilization and Embriology Authority).

Otro estándar de calidad es el seguimiento de las buenas prácticas de laboratorio FIV, establecidas por la ESHRE 5 (European Society of Human Reproduction and Embriology). Estas guías son muy importantes pero son inevitablemente subjetivas y son el reflejo de prácticas locales del país donde se han desarrollado.

La vigilancia sanitaria y el control de calidad de los productos y servicios que prestan las Unidades de Biomedicina Reproductiva en Colombia corresponde de manera exclusiva al Instituto de Vigilancia de Medicamentos y Alimentos, Invima, de conformidad con lo señalado por el articulo 245 de la ley 100 de 1993, según el decreto 2309 del 15 de Octubre del 2002. Nuestro centro reporta junto con 100 centros más a la Red Latino Americana de Reproducción Asistida.

\section{¿Cuáles son los indicadores de calidad en un centro de Infertilidad?}

- Tasa de embarazo por aspiración es la razón entre el número de pacientes grávidas por el número de aspiraciones realizadas.

- Tasa de parto por aspiración es la razón entre el número de partos atendidos por el número de aspiraciones realizadas.

- Tasa de parto con un recién nacido vivo por transferencia es la razón entre el número de partos con un recién nacido vivo por el número total de transferencias realizadas.

- Tasa de recién nacido vivo único a término por ciclo iniciado es la razón del número de un recién nacido vivo a término, por el número de ciclos iniciados.

- Tasa de parto único por embrión transferido es la razón entre el número de partos de un feto único por el número de transferencias de un solo embrión.

- Tasa de partos por embrión es la razón entre el número de partos por el número de embriones transferidos.

- Tasa de parto de un solo niño vivo es la razón entre el número de partos de un niño vivo por el número total de partos.

\section{DISCUSIÓN}

\section{¿Cuál es la tasa correcta de embarazo para FIV?}

La Red Latino Americana de Reproducción Asistida presenta la siguiente información de los diferentes centros que trabajan en técnicas de reproducción asistida (TRA) para el año $2002^{6}$ 
- Tasa de embarazo por aspiración: Para FIV, 30\%, y $31 \%$ para ICSI.

- Tasa de parto por aspiración: Para FIV 24,3\% y para ICSI 23,9\%; la tasa de embarazo múltiple se mantiene en niveles cercanos al 30,4\% (doble $22 \%$, triple o mas $7,7 \%)^{6}$

- La tasa de partos con (1) un recién nacido vivo por transferencia es de un $26 \%$, de esta un $18 \%$ corresponde a parto único, 6,5\% partos gemelares y $1,5 \%$ a partos triples. ${ }^{6}$

Las tasas antes descritas son bastante similares a las presentadas por el Centro de Control de Enfermedades (Centre for Disease Control CDC de Estados Unidos).

Sin embargo utilizar estas cifras para comparar programas debe hacerse con precaución debido a que se deben considerar los siguientes aspectos:

Las tasas más altas de embarazo no necesariamente hacen mejor a un centro de reproducción. Unas tasas de embarazo bajas, por ejemplo 10 a 15\% por ciclo pueden indicar un laboratorio pobre, pero igualmente puede reflejar un porcentaje alto de pacientes de mal pronóstico, como por ejemplo pacientes malas respondedoras. Pero si estas pacientes que no obtuvieron resultado en otro centro y que además tenían una FSH alta el día 3 del ciclo, se embarazan, entonces estas tasas del 10 al 15\% se pueden mirar como excelentes.

Por otra parte, tasas de embarazo del 50 al 70\% por ciclo, deberían inmediatamente suscitar preocupación ya que únicamente se estarían tratando pacientes de buen pronóstico. Las pacientes con menos posibilidades podrían ser excluidas. Esto sin embargo no es una buena práctica. ¿Se le debería negar la Fertilización in Vitro a una paciente que tenga un pronóstico menor que el promedio, digamos un 10 al 15\% por ciclo? Centros con tasas de embarazo mayores del $50 \%$ tienen que excluir a las pacientes con un bajo pronóstico si ellos quieren mantener sus altas tasas de éxito, por tanto esto no refleja una atención con calidad para esta población.

Los registros existentes en Estados Unidos, Europa y Latino América se establecieron inicialmente para medir la calidad entre los centros, lo contrario en realidad puede haber pasado. La atención enfocada únicamente en tasas de embarazo puede haber presionado a los centros de FIV a tener las más altas tasas de embarazo al precio que sea. Una revisión del registro nacional de Estados Unidos de América muestra que aquellos con tasas de embarazo por encima de $50 \%$ tienen una inaceptable tasa de gestación múltiple. ${ }^{8}$ La alta tasa de morbilidad y mortalidad neonatal es el precio que se paga cuando las tasas de embarazo son la primera prioridad para evaluar calidad.

La discusión sobre cuál es la medida apropiada para evaluar el éxito de la reproducción asistida sigue presente, Min y colaboradores de la Universidad de Monash en Australia ${ }^{9}$ puntualizaron que la medida más apropiada de éxito para la técnica de reproducción asistida (TRA) es el "nacimiento con vida de una gestación única a término por ciclo iniciado". Esta medida genera una desafortunada tasa de éxito del 11\%. No obstante la veracidad de esta cifra, muchas clínicas de infertilidad podrían ser renuentes a reportar esta tasa como un resultado final.

Información europea emitida por la ESHRE en el 2000, reporta una tasa de 15,2\% de "parto único por embrión trasferido". ${ }^{10}$ Esta nueva medida de éxito difiere de la medida australiana ya que el parámetro "término" no fue utilizado y "número de transferidos" sustituye a "ciclos iniciados". Otro parámetro de excelencia sugerido por la ESHRE es "número de partos por embrión".

Obviamente, este parámetro difiere de la tasa de implantación. Una tasa alta de implantación es deseable; sin embargo, puede creerse que un programa de TRA tenga una alta tasa de implantación si se han colocado tres embriones y da origen a una gestación triple. Al contrario, "la tasa parto por embrión transferido" hará que el número de embriones que transferimos sea menor.

Algunos autores manifiestan que la conclusión y recomendación hecha por la Sociedad Europea de Reproducción Humana y Embriología (ESHRE) ${ }^{12}$ de que únicamente se debía tener en cuenta los partos 
de un solo niño vivo como medida de éxito de un tratamiento de infertilidad, debería ser evaluada en términos de su efecto en cada pareja infértil y en la utilización de los recursos nacionales de salud.

La decisión de que el embarazo gemelar es una complicación y que el único resultado aceptable en el tratamiento de la infertilidad es el nacimiento de un sólo niño vivo, está fundamentada en una información incompleta, además es superflua, e indiferente para la pareja que requiere de técnicas de reproducción asistida (TRA) o inducción de ovulación (IO) para conseguir un embarazo. ${ }^{13}$

Los embarazos múltiples pueden ser la manifestación azarosa del tratamiento moderno de la infertilidad, los problemas asociados con el nacimiento de gemelares, el cual es un fenómeno natural, son mínimos comparados con las complicaciones que se presentan en los embarazos triples o de un número mayor.

En el año 2000, la fertilización in Vitro (FIV) y otras técnicas de reproducción asistida fueron responsables en Estados Unidos de un 12\% de 118.997 niños nacidos como gemelares comparado con un 42\% de 7.328 nacimientos de triples o de un número mayor. ${ }^{14}$ Durante el mismo año, la inducción de la ovulación sin utilizar técnicas de reproducción asistida fue responsable por un $21 \%$ de nacimientos gemelares y de un $40 \%$ de nacimientos de triples o más, mientras que se estimó que el 67\% de nacimientos gemelares y $18 \%$ de nacimientos de triples o más fueron resultado de embarazos naturales. ${ }^{14}$ Limitando el número de embriones a transferir a uno solo reduciría el número de nacimientos triples o más de todas las causas en un $42 \%$ y podría reducir el número de nacimientos gemelares en no mas del $12 \%$, algunos casos de nacimientos gemelares debido a FIV pueden ocurrir por división del zigoto. Limitando él numero de embriones trasferidos a dos, podría eliminarse también el nacimiento de triples en un $42 \%$ y el nacimiento de embarazos gemelares debido a FIV menos del 20\%, por la eliminación de nacimientos gemelares que comenzaron como una gestación de triples o más.
Claramente, la transferencia de dos embriones podría reducir el número de nacimientos múltiples, lo cual tiene un alto riesgo de complicaciones, en una forma tan efectiva como la transferencia de un solo embrión. ${ }^{13}$

Hay evidencia inequívoca en cuanto a tasas de embarazo que al transferir un solo embrión o transferir dos, esta es de 11,3\% cuando se trasfiere un solo embrión y $37,2 \%$ cuando se trasfieren dos embriones. ${ }^{15-16}$

Satisfacción del cliente; Numerosos estudios realizados sobre la satisfacción de pacientes con los servicios de FIV han aportado importantes enseñanzas a los proveedores de estos servicios. Por ejemplo, mientras la mayoría de los pacientes están generalmente felices con su tratamiento, pacientes estudiados en Estados Unidos ${ }^{17-19}$ expresaron su deseo de una mayor información, específicamente de forma escrita.

\section{Cómo se debe definir la calidad en un centro de FIV}

En la estructuración para definir medidas de calidad debemos determinar nuestro producto.

Nuestro producto es servicio, ayudar a las parejas infértiles a manejar su problema. Algunas veces tenemos éxito, algunas veces fallamos. Pero calidad en el contexto de un programa de FIV significa la calidad del servicio que damos a las parejas infértiles que se nos aproximan buscando ayuda. De acuerdo con lo anterior, aplicamos ocho principios de gestión de calidad que constituyen la base de las normas de sistema de gestión de calidad de la familia de Normas ISO 9000, que fueron utilizadas por la dirección del centro con el fin conducir a la organización hacia una mejora en el desempeño y la satisfacción de la pareja infértil. ${ }^{20}$

1. Enfoque al cliente/paciente: comprender sus necesidades actuales y futuras, satisfacer sus requisitos y esforzarse en exceder sus expectativas.

2. Dirección: La cual establece una unidad de propósito con la cual se comprometan todos los miembros de la organización. 
3. Participación del personal. El personal, a todos los niveles con su total compromiso posibilita que sus habilidades sean utilizadas para el beneficio de la organización.

4. Enfoque basado en procesos: las actividades y los recursos relacionados se gestionan como un proceso.

5. Enfoque de sistema para la gestión: identificar, entender y gestionar los procesos interrelacionados como un sistema, esto contribuye a la eficacia y eficiencia de la organización para cumplir sus objetivos.

6. Mejora continua: la mejora continua del desempeño global de la organización es un objetivo permanente de ésta.

7. Enfoque basado en hechos para la toma de decisiones: Las decisiones eficaces se basan en análisis de los datos y la información.

8. Relaciones mutuamente beneficiosas con el proveedor. La organización y sus proveedores son interdependientes, buscando una relación mutua beneficiosa.

\section{Direccionamiento Estratégico en FECUNDAR}

Definición de la misión, visión y de una política de calidad establecimiento de procesos de valor agregado en la consulta de Infertilidad, en procesos de técnicas de reproducción asistida (inducción de ovulación, embriones a transferir), métodos de laboratorio, educación médica continua, procesos de gestión administrativa, administración de calidad/auditorías y satisfacción del paciente.

Nuestros resultados de acuerdo con los indicadores establecidos para este proceso son:

Número Embarazos por Tratamientos Realizados: FIV en $\leq 35$ años 28\%, FIV en 35-39 años $20 \%$, FIV con óvulo donado 30\%, ICSI en $\leq 35$ años 28\%, ICSI entre 35 -39 años 18\%, ICSI con óvulo donado $30 \%$, IIU $\leq 35$ años 20\%, IIU >35 años $18 \%$.

\section{CONCLUSIONES}

Queremos poner en consideración la experiencia que hemos tenido al introducir los estándares de calidad de la norma de ISO 9000 en nuestro centro para el manejo de los problemas de fertilidad, tanto a pacientes como a los demás centros que dan este servicio en Colombia. Estos estándares internacionales, de aplicación mundial definirán mejor los resultados que son importantes para nuestros pacientes y crearán los mecanismos para el mejoramiento del cuidado que nosotros proporcionamos. La adaptación de un estándar internacional para el tratamiento de la infertilidad tiene la gran ventaja de ser capaz de unificar pautas nacionales y desarrollar un consenso sobre lo que son las medidas importantes de calidad en nuestro campo.

Los beneficios internos de ISO 9000 son la documentación de los procesos, una mayor conciencia en la calidad por parte de los empleados de la institución, cambios en la cultura organizacional que resultan en una mayor productividad y la instalación de un sistema general de calidad. Los beneficios externos son la ventaja que se consigue sobre los competidores no certificados, el cumplimiento de los requerimientos de los clientes, una calidad percibida más alta y probablemente una mayor satisfacción del cliente. ${ }^{2}$

\section{REFERENCIAS}

1. Wickland M, Sjoblom C. The application of quality systems in ART programs. Mol Cell Endocrinol 2000;166:3-7

2. Koontz H, Weihrich H. Administración global, comparada y de calidad. En: Koont M, Weihrich H. Administración: Una perspectiva global. 11a Edición México: McGraw-Hill Interamericana; 1998. p. 81-120.

3. Lock D, Smith DJ. Gower Handbook of Quality Management. Aldershot, GB: Gower Publishing; 1997. p. 2-44.

4. Lass A, Brinsden P. How do patients choose private in vitro fertilization treatment? A customer survey in tertiary fertility center in the United Kingdom. Fertil Steril 2001;75:893-7. 
5. Gianaroli L, Plachot M, van Kooij R, Al-Hasani S, Dawson K, DeVos A, et al. ESHRE guidelines for good practice in IVF laboratories. Committee of the Special Interest Group on Embryology of the European Society of Human Reproduction and Embriology. Hum Reprod 2000;15:2241-6.

6. Franco JG Jr. Registro Latino Americano de Reproducción Asistida. 2002.

7. Schieve LA, Reynolds MA. What is the most relevant standard of success in assisted reproduction? Challenges in measuring and reporting success rates for assisted reproductive technology treatments: what is optimal? Hum Reprod 2004; 19:778-82.

8. Society for Assisted Reproductive Technology and American Society for Reproductive Medicine. Assisted reproductive technology in the U.S: 1997 results generated from American Society of Reproductive Medicine/Society for Assisted Reproductive Technology. Fertil Steril 2000;74:641-53.

9. Min JK, Han KY, Kim EC, Kim YM, Lee SW, Kim OH, et al. What is the most relevant standard of success in assisted reproduction? The singleton, term gestation, lives birth rate per cycle initiated: the BESST endpoint for assisted reproduction. Hum Reprod 2004;19:3-7.

10. Nyboe Andersen A, Gianaroli L, Nygren KG; European IVF-monitoring programme; European Society of Human Reproduction and Embryology. Assisted reproductive technology in Europe, 2000. Results generated from European registers by ESHRE. Hum Reprod 2004;19:490-503.

11. Nygren KG, Andersen AN. Assisted reproductive technology in Europe, 1999. Results generated from European registers by ESHRE. Hum Reprod 2002;17:3260-74.
12. Land JA, Evers JL. Risks and complications in assisted reproductions techniques: Report of and ESHRE consensus meeting. Hum Reprod 2003;18:455-7.

13. Dickey RP, Sartor BM, Pyrzak R. What is the most relevant standard of success in assisted reproduction? No single outcome measure is satisfactory when evaluating success in assisted reproduction; both twin births should be counted as successes. Hum Reprod 2004;19:783-7.

14. Reynolds MA, Schieve LA, Martin JA, Jeng G, Macaluso M. Trends in multiple births conceived using assisted reproductive technology, United States, 1997-2000. Pediatrics 2003;111:1159-62.

15. Centers for Disease Control and Prevention, American Society for Reproductive Medicine/ Society of Assisted Reproductive Technology and Resolve: The National Infertility Association (2003) 2001 Assisted Reproductive Technology Success Rates. Department of Health and Human Services, Centers for Disease Control and Prevention, Atlanta, December 2003

16. Pandian Z, Templeton A, Serour G, Bhattacharya S. Number of embryos for transfers after IVF and ICSI a Cochrane review. Hum Reprod 2005;10:2681-7.

17. Halman LJ, Abbey A, Andrews FM. Why are couples satisfied with infertility treatment? Fertil Steril 1993;59:1046-54.

18. Souter VL, Penney G, Hopton JL, Templeton AA. Patient satisfaction with the management of infertility. Human Reprod 1998;13:1831- 6.

19. Hammarberg K, Astbury J, Baker H. Women's experience of IVF: a follow-up study. Hum Reprod 2001;16:374-83.

20. ICONTEC 2002, Norma Técnica Colombiana NTCISO 9000: Sistema de Gestión de Calidad 